\title{
Review of: "Multiple soil map comparison highlights challenges for predicting topsoil organic carbon concentration at national scale"
}

\author{
Chun Liu ${ }^{1}$ \\ 1 Jinan University
}

Potential competing interests: The author(s) declared that no potential competing interests exist.

This study was very interesting and well organized. It was useful and provide a novel method for better accurately estimating the soil organic carbon storage and budget at national scale. In my opinion, the only major concern was that "how did authors define the depth of topsoil? 0-15 cm depth? 0-20cm depth? 0$30 \mathrm{~cm}$ depth? " The author should make it more clearly for readers. Another is the Grid resolution for eight maps. The precision of SOC data might exist bias that obtained from different grid resolution of maps. Please clarify it more clearly for readers. 